\title{
Tradeoff between extractable mechanical work, accessible entanglement, and ability to act as a reference system, under arbitrary superselection rules
}

\author{
J.A. Vaccaro, ${ }^{1,2}$ F. Anselmi, ${ }^{3,2}$ H.M. Wiseman, ${ }^{1}$ and K. Jacobs ${ }^{1,4}$ \\ ${ }^{1}$ Centre for Quantum Dynamics, Griffith University, Brisbane, 4111 Australia \\ ${ }^{2}$ Quantum Physics Group, University of Hertfordshire, Hatfield, AL10 9AB, UK \\ ${ }^{3}$ Venetian Institute of Molecular Medicine, Via Giuseppe Orus, 235129 Padova, Italy \\ ${ }^{4}$ Department of Physics, University of Massachusetts at Boston, 100 Morrissey Blvd, Boston, MA 02125, USA
}

(Dated: January 10, 2008)

\begin{abstract}
Superselection rules (SSRs) limit the mechanical and quantum processing resources represented by quantum states. However SSRs can be violated using reference systems to break the underlying symmetry. We show that there is a duality between the ability of a system to do mechanical work and to act as a reference system. Further, for a bipartite system in a globally symmetric pure state, we find a triality between the system's ability to do local mechanical work, its ability to do "logical work" due to its accessible entanglement, and its ability to act as a shared reference system.
\end{abstract}

PACS numbers: 03.67.Mn, 02.20.-a, 03.65.Fd, 03.65.Ta

\section{INTRODUCTION}

Global conservation laws give rise to superselection rules (SSRs) which forbid the observation of coherences between particular subspaces of states $[1,2]$. Such global laws do not apply in subsystems $[2,3]$. For example, the angular momentum of an object can be changed provided the total angular momentum of the object and another system, the ancilla, is conserved. The ancilla here acts as a reference system which alleviates the affect of the SSR by locally breaking the associated symmetry [2]. Conversely, the lack of a reference system induces the SSR. For example, without a spatial orientation frame, the state of a spin- $\frac{1}{2}$ particle will be completely mixed.

The last few years has witnessed a resurgence of interest in SSRs and quantum reference systems particularly within the context of quantum information theory. The recent review by Bartlett, Rudolph and Spekkens [4] describes the current state of affairs. For example, Eisert et al. [5] and recently Jones et al. [6] studied the decrease in distillable entanglement due to the loss of relative-ordering information for sets of ebits. The optimal cost of aligning reference frames has been calculated in a number of different settings [7]. Communication in the presence or absence of shared reference frames has been extensively studied by Bartlett et al. [8]. The conservation of particle number was shown by two of us [9] to limit shared particle entanglement. The repercussions for various systems including those in condensed matter physics was explored by Dowling et al. [10]. This constraint on shared entanglement of particles has been generalized to arbitrary SSRs by Bartlett and Wiseman [11]. For the special case of a U(1)-SSR, a new resource, the shared phase reference, has been studied by Vaccaro et al. [12], and quantified in the asymptotic [13] and nonasymptotic [14] regimes.

In this paper we investigate the effect of a SSR on the resources represented by a quantum state. Following Oppenheim et al. we quantify the resources in terms of mechanical work extractable from a heat bath and logical work as performed in quantum information processing (QIP) [15]. We expose a fundamental tradeoff between the extractable work under the SSR and the ability to act as a reference system for the SSR. We treat both the unipartite and the bipartite case. The latter shows a triality between the accessible entanglement, locally extractable mechanical work and the ability to act as a shared reference system. These results are crucial for fully understanding and quantifying resources used in QIP.

We wish to emphasize from the outset that the resources are determined in the non-asymptotic regime in the following sense. While the asymptotic limit $\rho^{\otimes n}$ for $n \rightarrow \infty$ is often taken when studying resources such as entanglement, this limit is not appropriate for the problems addressed here. Indeed, in the asymptotic limit reference systems such as those for spatial orientation and quantum phase reduce to their less-interesting classical counterparts. Instead the situation we consider is when the resources such as accessible entanglement, local work and reference ability are measured for just one copy of the state $\rho$. The same situation has been treated in previous work $[9,12]$ for the specific case of the accessible entanglement of indistinguishable particles. In operational terms, we imagine that the state of the system is transferred by operations that are allowed by the SSR to ancillary systems which are not themselves subject to the SSR. Once transferred to the SSR-free ancillas, the resources are fungible in the sense that they can be used, processed, transferred etc. in a manner free of the SSR. Our results quantify the amount of the resources that are transferable in this way from the single copy of the state $\rho$ and made SSR free. This is what we mean by the terms extractable work and accessible entanglement. Thereafter one could consider the asymptotic limit of the resources contained in the SSR-free ancillary systems and this would justify the entropic measures for work and entanglement. 


\section{EXTRACTABLE WORK AND ASYMMETRY}

\section{A. Framework for the SSR}

An SSR is associated with a set $\tau=\{T(g)\}$ of unitary operators indexed by $g$ whose effect on the system is undetectable. There are two physically-motivated conditions on the set $\tau$. If the effect of an operator $T(g)$ is not detectable, then so is the effect of the time-reversed process which is given by the inverse $T^{-1}(g)$. This means if $T(g) \in \tau$ then $T^{-1}(g) \in \tau$. If the effects of two operators $T\left(g_{1}\right)$ and $T\left(g_{2}\right)$ are not detectable then the effect of their product $T\left(g_{1}\right) T\left(g_{2}\right)$ is also not detectable. This means that if $T\left(g_{1}\right) \in \tau$ and $T\left(g_{2}\right) \in \tau$ then $T\left(g_{1}\right) T\left(g_{2}\right) \in \tau$. Thus the set $\tau$ is closed under multiplication. These conditions endow $\tau$ with a group structure, i.e. the set

$$
\tau=\{T(g): g \in G\}
$$

is a unitary representation of the abstract group $G=\{g\}$. We shall label the SSR associated with group $G$ as $G$-SSR [16].

Let $\rho$ be an arbitrary density operator representing the (possibly mixed) state of a system. A $G$-SSR restricts not this state, but rather the allowed operations on it to those that are $G$-invariant [11]. That is, an allowed operation $\mathcal{O}$ must satisfy

$$
\mathcal{O}\left[T(g) \rho T^{\dagger}(g)\right]=T(g)(\mathcal{O} \rho) T^{\dagger}(g), \forall g \in G .
$$

Under this restriction, our effective knowledge of the system is represented not by $\rho$ but by the "twirl" of $\rho[11]$

$$
\mathcal{G}_{G}[\rho] \equiv \frac{1}{|G|} \sum_{g \in G} T(g) \rho T^{\dagger}(g)
$$

where $|G|$ is the order of the group $G$.

We will require that the representation factorizes as

$$
T(g)=T_{1}(g) \otimes T_{2}(g) \otimes \cdots
$$

for multipartite systems whose corresponding Hilbert space is given by $\mathcal{H}=\mathcal{H}_{1} \otimes \mathcal{H}_{2} \otimes \cdots$ where $\mathcal{H}_{n}$ is the Hilbert space for the system labeled by $n$.

\section{B. Extractable work}

The purpose of a reference system is to mask the effects of the $G$-SSR by yielding less mixing than given in Eq. (2.3). A physically meaningful definition of the ability of a system to act as a reference system should therefore be based on a physical quantity that measures a state's mixedness. This measure is conveniently provided by the amount of mechanical work that can be extracted from a thermal reservoir at temperature $T$ using quantum state $\rho$. This is given by $[15,18]$

$$
W(\rho)=k_{\mathrm{B}} T[\log D-S(\rho)],
$$

where $D$ is the dimension of the Hilbert space and

$$
S(\rho) \equiv-\operatorname{Tr}[\rho \log \rho]
$$

is the von Neumann entropy of $\rho$. This expression shows that the more pure the state $\rho$ is, the more work that can be extracted using it. For convenience, in the following we set $k_{\mathrm{B}} T=1$ and use the binary logarithm. In the presence of the $G$-SSR this resource reduces to the extractable work

$$
W_{G}(\rho) \equiv W\left(\mathcal{G}_{G}[\rho]\right) .
$$

The proof follows the same lines as that of Ref. [11] for accessible entanglement. The crucial point here is that once the work $W_{G}(\rho)$ has been extracted by a $G$-invariant operation, applying $\mathcal{G}$ to the system does not change the amount of work that was extracted. According to Eq. (2.2), the same result is obtained if $\mathcal{G}$ is applied to the system before the work is extracted, and so the extractable work is $W\left(\mathcal{G}_{G}[\rho]\right)$. A symmetric state, i.e. one for which

$$
\mathcal{G}_{G}[\rho]=\rho,
$$

suffers no loss in its ability to do work. In contrast, the extractable work possible for asymmetric states $\left(\mathcal{G}_{G}[\rho] \neq\right.$ $\rho$ ), is reduced under the $G$-SSR.

As an example, consider a spin- $\frac{1}{2}$ particle prepared in state $\rho$ by Alice and sent to Bob, and let Bob have knowledge only of the direction of Alice's $z$ axis. Bob cannot distinguish rotations by Alice about the $z$ axis. Thus his knowledge of the state is constrained by the SSR induced by the $U(1)$ symmetry group associated with the unitary representation

$$
\left\{T(\theta): \theta \in[0,2 \pi), T(\theta)=\exp \left(i \theta J_{z} / \hbar\right)\right\}
$$

where $J_{z}=\frac{\hbar}{2} \sigma_{z}$ and $\sigma_{z}$ is the Pauli operator for the $z$ component of spin. Accordingly Bob ascribes the state

$$
\mathcal{G}_{U}[\rho]=\frac{1}{2 \pi} \int_{0}^{2 \pi} e^{i \theta J_{z} / \hbar} \rho e^{-i \theta J_{z} / \hbar} d \theta
$$

to the spin. Consider the spin-up state $\rho=|1\rangle\langle 1|$, where $\sigma_{z}| \pm 1\rangle= \pm| \pm 1\rangle$.

The state $|1\rangle$ is symmetric with respect to $\{T(\theta)\}$ so for this state $W=1$ and the amount of extractable work is also $W_{U}=1$. In contrast, the state $|+\rangle=(|1\rangle+|-1\rangle) / \sqrt{2}$ is asymmetric with respect to $\{T(\theta)\}$, with

$$
\mathcal{G}_{U}[\rho]=\frac{1}{2}(|1\rangle\langle 1|+|-1\rangle\langle-1|) .
$$

Even though the state $|+\rangle$ has $W=1$, under the SSR Bob can extract no work as $W_{U}=0$.

\section{Asymmetry}

A SSR thus introduces the need for a new resource: a system acting as a reference system to break the underlying symmetry. We now show that [17]

$$
A_{G}(\rho) \equiv S\left(\mathcal{G}_{G}[\rho]\right)-S(\rho),
$$


which is the natural entropic measure of the asymmetry of $\rho$ with respect to $G$, is a measure that quantifies the ability of a system to act as a reference system. To do this we need to show that $A_{G}$ has the following properties:

(i) $A_{G}(\rho) \geq 0$;

(ii) $A_{G}(\rho)=0$ iff $\rho$ is symmetric;

(iii) $A_{G}(\rho)$ cannot increase under the restriction of the $G$-SSR; and

(iv) $A_{G}(\rho)$ quantifies the ability of $\rho$ to act as a reference system.

The first two follow directly from the properties of the entropy function [19]. For the third, we have

Theorem 1. No G-invariant operation can increase (on average) the asymmetry $A_{G}(\rho)$ of a state $\rho$.

Proof. The most general $G$-invariant operation is a measurement that transforms an initial state $\rho$ into one of $M$ states

$$
\rho_{j}=\frac{1}{P_{j}} \mathcal{O}_{j}[\rho]
$$

such that

$$
\mathcal{O}_{j}\left[T(g) \rho T^{\dagger}(g)\right]=T(g)\left(\mathcal{O}_{j}[\rho]\right) T^{\dagger}(g), \forall g \in G,
$$

with probability $P_{j}=\operatorname{Tr}\left(\mathcal{O}_{j}[\rho]\right)$. This operation includes the possibility of adding ancillas in prepared states and performing unitary operations and measurements on the combined system and ancillas. We wish to show that

$$
A_{G}(\rho) \geq \sum_{j} P_{j} A_{G}\left(\rho_{j}\right)
$$

i.e. from Eq. (2.12)

$$
S\left(\mathcal{G}_{G}[\rho]\right)-S(\rho) \geq \sum_{j} P_{j}\left[S\left(\mathcal{G}_{G}\left[\rho_{j}\right]\right)-S\left(\rho_{j}\right)\right],
$$

which can be rearranged as

$$
S\left(\mathcal{G}_{G}[\rho]\right)-\sum_{j} P_{j} S\left(\mathcal{G}_{G}\left[\rho_{j}\right]\right) \geq S(\rho)-\sum_{j} P_{j} S\left(\rho_{j}\right) .
$$

Note that, because the $\mathcal{O}_{j}$ are $G$-invariant, we can interchange the order of the twirl $\mathcal{G}_{G}$ and the $\mathcal{O}_{j}$ operations. Denoting the average change in entropy under the measurement operation by

$$
\left\langle\Delta S_{\mathcal{O}}(\rho)\right\rangle=S(\rho)-\sum_{j} P_{j} S\left(\rho_{j}\right)
$$

allows us to rewrite the inequality we wish to prove as

$$
\left\langle\Delta S_{\mathcal{O}}\left(\mathcal{G}_{G}[\rho]\right)\right\rangle \geq\left\langle\Delta S_{\mathcal{O}}(\rho)\right\rangle .
$$

We now use the following three facts: (i) for all operations the average entropy reduction, $\left\langle\Delta S_{\mathcal{O}}(\rho)\right\rangle$, is concave in $\rho[20]$, and so, e.g. $\left\langle\Delta S_{\mathcal{O}}\left(\sum_{j} p_{j} \rho_{j}\right)\right\rangle \geq \sum_{j} p_{j}\left\langle\Delta S_{\mathcal{O}}\left(\rho_{j}\right)\right\rangle ;$

(ii) the twirl operation produces the convex mixture

$$
\mathcal{G}_{G}[\rho]=\frac{1}{|G|} \sum_{g} \sigma_{g}
$$

where $\sigma_{g}=T(g) \rho T^{\dagger}(g)$; and

(iii) $\left\langle\Delta S_{\mathcal{O}}\left(\sigma_{g}\right)\right\rangle=\left\langle\Delta S_{\mathcal{O}}(\rho)\right\rangle$ for all $g$ due to the $G$ invariance of the $\mathcal{O}_{j}$ and the unitarity of the $T(g)$.

Putting these together we have

$$
\begin{aligned}
\left\langle\Delta S_{\mathcal{O}}\left(\mathcal{G}_{G}[\rho]\right)\right\rangle & =\left\langle\Delta S_{\mathcal{O}}\left(\frac{1}{|G|} \sum_{g} \sigma_{g}\right)\right\rangle \\
& \geq \frac{1}{|G|} \sum_{g}\left\langle\Delta S_{\mathcal{O}}\left(\sigma_{g}\right)\right\rangle \\
& =\frac{1}{|G|} \sum_{g}\left\langle\Delta S_{\mathcal{O}}(\rho)\right\rangle=\left\langle\Delta S_{\mathcal{O}}(\rho)\right\rangle
\end{aligned}
$$

which completes the proof of Eq. (2.19).

To show the fourth property let us first define $\Upsilon\left(X ; \rho_{1}, \rho_{2}\right)$, the synergy of a quantity $X$, as

$$
\Upsilon\left(X ; \rho_{1}, \rho_{2}\right) \equiv X\left(\rho_{1} \otimes \rho_{2}\right)-\left[X\left(\rho_{1}\right)+X\left(\rho_{2}\right)\right]
$$

for two systems in states $\rho_{1}$ and $\rho_{2}$. The extent to which system 1 acts as a reference system for system 2 (or vice versa) is the synergy of the extractable work, $\Upsilon\left(W_{G} ; \rho_{1}, \rho_{2}\right)$; that is, the amount by which the extractable work of the whole is larger than the sum of the extractable work of the parts. Then we have the following:

Theorem 2. The synergy of the extractable work is bounded by asymmetry:

$$
\Upsilon\left(W_{G} ; \rho_{1}, \rho_{2}\right) \leq \min \left\{A_{G}\left(\rho_{1}\right), A_{G}\left(\rho_{2}\right)\right\}
$$

where $\rho_{1}$ and $\rho_{2}$ are arbitrary states of two systems sharing the same symmetry group $G$. Further, this bound is achievable, in the sense that for every $\rho_{1}$ there exists a $\rho_{2}$ such that $\Upsilon\left(W_{G} ; \rho_{1}, \rho_{2}\right)=A_{G}\left(\rho_{1}\right)$.

Proof. We first note from Eqs. (2.5), (2.7) and (2.12) that the extractable work can be written as

$$
W_{G}(\rho)=W(\rho)-A_{G}(\rho)
$$

and, because $W\left(\rho_{1} \otimes \rho_{2}\right)=W\left(\rho_{1}\right)+W\left(\rho_{2}\right)$, the synergy of the extractable work may be written as

$$
\begin{aligned}
& \Upsilon\left(W_{G} ; \rho_{1}, \rho_{2}\right) \\
& =W_{G}\left(\rho_{1} \otimes \rho_{2}\right)-\left[W_{G}\left(\rho_{1}\right)+W_{G}\left(\rho_{2}\right)\right] \\
& =\left[W\left(\rho_{1} \otimes \rho_{2}\right)-A_{G}\left(\rho_{1} \otimes \rho_{2}\right)\right] \\
& \quad \quad-\left[W\left(\rho_{1}\right)-A_{G}\left(\rho_{1}\right)+W\left(\rho_{2}\right)-A_{G}\left(\rho_{2}\right)\right] \\
& =A_{G}\left(\rho_{1}\right)+A_{G}\left(\rho_{2}\right)-A_{G}\left(\rho_{1} \otimes \rho_{2}\right) .
\end{aligned}
$$


We next note that $A_{G}\left(\rho_{1} \otimes \rho_{2}\right)$ is equal to the Holevo $\chi$ quantity [19], $\chi_{12}$, for the ensemble

$$
\left\{\left(P_{g}, \sigma_{g}\right) \forall g \in G\right\}
$$

where $P_{g}=|G|^{-1}$ is the probability associated with the state $\sigma_{g}$ and

$$
\sigma_{g}=\left[T(g) \rho_{1} T(g)^{\dagger}\right] \otimes\left[T(g) \rho_{2} T(g)^{\dagger}\right] .
$$

Similarly, the Holevo $\chi$ for the ensemble traced over subsystem 2 or 1 is $\chi_{1}=A_{G}\left(\rho_{1}\right)$ or $\chi_{2}=A_{G}\left(\rho_{2}\right)$, respectively. The Holevo $\chi$ is non-increasing under partial trace [19], so

$$
A_{G}\left(\rho_{1} \otimes \rho_{2}\right) \geq A_{G}\left(\rho_{j}\right) \text { for } j=1,2 .
$$

Applying this to Eq. (2.25) gives the desired result.

To show achievability, choose $\rho_{2}=|\psi\rangle\langle\psi|$ such that $\left\langle\psi_{g^{\prime}} \mid \psi_{g}\right\rangle=\delta_{g, g^{\prime}}$ where $\left|\psi_{g}\right\rangle \equiv T_{2}(g)|\psi\rangle$. For finite groups this can be done with a normalisable state $\rho_{2}$ whereas for Lie groups one can choose a normalisable state on a subspace of sufficiently large dimension [3]. Then using Eqs. (2.3) and (2.4) we have

$$
\begin{aligned}
\mathcal{G}_{G} & {\left.\left[\rho_{1} \otimes \rho_{2}\right]\right) } \\
& =\frac{1}{|G|} \sum_{g \in G}\left[T_{1}(g) \otimes T_{2}(g)\right] \rho_{1} \otimes \rho_{2}\left[T_{1}^{\dagger}(g) \otimes T_{2}^{\dagger}(g)\right] \\
& =\frac{1}{|G|} \sum_{g \in G}\left[T_{1}(g) \rho_{1} T_{1}^{\dagger}(g)\right] \otimes\left|\psi_{g}\right\rangle\left\langle\psi_{g}\right|
\end{aligned}
$$

The orthonormality of the set $\left\{\left|\psi_{g}\right\rangle: g \in G\right\}$ ensures that

$$
\begin{aligned}
S\left(\mathcal{G}_{G}\left[\rho_{1} \otimes \rho_{2}\right]\right) & =\sum_{g \in G} \frac{1}{|G|}\left\{S\left[T_{1}(g) \rho_{1} T_{1}^{\dagger}(g)\right]-\log \left(\frac{1}{|G|}\right)\right\} \\
& =S\left(\rho_{1}\right)+S\left(\mathcal{G}_{G}\left[\rho_{2}\right]\right)
\end{aligned}
$$

where we have used $S_{G}\left(\rho_{2}\right)=\log (|G|)$. Finally, using this result with Eqs. (2.12) and (2.25) and noting that $S\left(\rho_{1} \otimes \rho_{2}\right)=S\left(\rho_{1}\right)+S\left(\rho_{2}\right)$ shows

$$
\begin{aligned}
\Upsilon( & \left.W_{G} ; \rho_{1}, \rho_{2}\right) \\
= & \left\{S\left(\mathcal{G}_{G}\left[\rho_{1}\right]\right)-S\left(\rho_{1}\right)\right\}+\left\{S\left(\mathcal{G}_{G}\left[\rho_{2}\right]\right)-S\left(\rho_{2}\right)\right\} \\
& \quad-\left\{S\left(\mathcal{G}_{G}\left[\rho_{1} \otimes \rho_{2}\right]\right)-S\left(\rho_{1} \otimes \rho_{2}\right)\right\} \\
= & \left.S\left(\mathcal{G}_{G}\left[\rho_{1}\right]\right)+S\left(\mathcal{G}_{G}\left[\rho_{2}\right]\right)-S\left(\mathcal{G}_{G} \rho_{1} \otimes \rho_{2}\right]\right) \\
= & S\left(\mathcal{G}_{G}\left[\rho_{1}\right]\right)-S\left(\rho_{1}\right) \\
= & A_{G}\left(\rho_{1}\right)
\end{aligned}
$$

which completes the proof of achievability.

To illustrate the phenomenon of synergy, consider the previous spin- $\frac{1}{2}$ example but now with two spins in the state $|+\rangle$. That is, Alice sends to Bob the state $\rho_{1} \otimes \rho_{2}$, with $\rho_{i}=|+\rangle\langle+|$ for $i=1,2$. Bob again assigns the state

$$
\mathcal{G}_{U}\left[\rho_{1} \otimes \rho_{2}\right]=\frac{1}{2 \pi} \int_{0}^{2 \pi} e^{i \theta J_{z} / \hbar}\left(\rho_{1} \otimes \rho_{2}\right) e^{-i \theta J_{z} / \hbar} d \theta,
$$

but now

$$
J_{z}=J_{z}^{(1)} \otimes I^{(2)}+I^{(1)} \otimes J_{z}^{(2)}
$$

where $I^{i}$ is the identity operator for system $i$. We find

$$
\mathcal{G}_{U}\left[\rho_{1} \otimes \rho_{2}\right]=\uplus \frac{1}{2}|1,1\rangle \uplus \frac{1}{2}(|1,-1\rangle+|-1,1\rangle) \uplus \frac{1}{2}|-1,-1\rangle .
$$

Here, for clarity, we have used the following notational convention which was introduced in Ref. [6]: $\uplus|\psi\rangle$ is to be read as $+|\psi\rangle\langle\psi|$. Thus, for example,

$$
\uplus \alpha|\psi\rangle \uplus \beta|\phi\rangle \equiv|\alpha|^{2}|\psi\rangle\left\langle\left.\psi|+| \beta\right|^{2} \mid \phi\right\rangle\langle\phi| \text {. }
$$

As before, $W\left(\rho_{i}\right)=1, W_{U}\left(\rho_{i}\right)=0$, and $A_{U}\left(\rho_{i}\right)=1$. But for the two spins together, $W\left(\rho_{1} \otimes \rho_{2}\right)=2, W_{U}\left(\rho_{1} \otimes \rho_{2}\right)=$ $\frac{1}{2}$, and $A_{U}\left(\rho_{1} \otimes \rho_{2}\right)=\frac{3}{2}$. Thus the synergy is

$$
\Upsilon\left(W_{U} ; \rho_{1}, \rho_{2}\right)=\frac{1}{2}>0
$$

One spin acts as a reference for the other and partially breaks this $U(1)-\mathrm{SSR}$. Notice that the work synergy is less than the asymmetries of the individual systems, $\Upsilon\left(W_{U} ; \rho_{1}, \rho_{2}\right)<A_{U}\left(\rho_{i}\right)=1$, in accord with Theorem 2.

Having established the significance of $A_{G}(\rho)$ for indicating the ability of a system to act as a $G$-reference system, we now observe that Eq. (2.24) represents a tradeoff or duality between this ability and the amount of work that can be extracted under the $G$-SSR:

$$
W(\rho)=W_{G}(\rho)+A_{G}(\rho) .
$$

That is, under the $G$-SSR, the extractable work $W(\rho)$ represented by a given state is split into two new resources, the extractable work $W_{G}$ and the asymmetry $A_{G}$.

\section{EXTENSION TO BIPARTITE SYSTEMS}

\section{A. Global and local SSRs}

Consider a system shared by two parties, Alice and Bob, such that the unitary representation of $G$ factorizes according to:

$$
T(g)=T_{\mathrm{A}}(g) \otimes T_{\mathrm{B}}(g) \forall g \in G .
$$

There are two ways the $G$-SSR operates on the bipartite system, globally and locally. They can be illustrated by considering their effect on the system state $\rho$. The global $G$-SSR acts when we have access to the whole system using either non-local operations or transporting the whole system to one site. Thus in direct accord with Eq. (2.3) for the uni-partite case, our effective knowledge 
of the system under the global $G$-SSR is not $\rho$ but

$$
\begin{aligned}
\mathcal{G}_{G}[\rho] & =\frac{1}{|G|} \sum_{g \in G} T(g) \rho T^{\dagger}(g) \\
& =\frac{1}{|G|} \sum_{g \in G} T_{\mathrm{A}}(g) \otimes T_{\mathrm{B}}(g) \rho T_{\mathrm{A}}^{\dagger}(g) \otimes T_{\mathrm{B}}^{\dagger}(g) .
\end{aligned}
$$

In contrast, each party $\mathrm{A}$ and $\mathrm{B}$ has access only to the part of the system at their respective site. Accordingly the $G$-SSR restricts their knowledge of the system to

$\mathcal{G}_{G \otimes G}[\rho]=\frac{1}{|G|^{2}} \sum_{g \in G} \sum_{g^{\prime} \in G} T_{\mathrm{A}}(g) \otimes T_{\mathrm{B}}\left(g^{\prime}\right) \rho T_{\mathrm{A}}^{\dagger}(g) \otimes T_{\mathrm{B}}^{\dagger}\left(g^{\prime}\right)$.

We use the tensor product operator in the symbol $\mathcal{G}_{G \otimes G}$ to indicate that the twirl operation acts locally on systems $\mathrm{A}$ and $\mathrm{B}$; this is manifest in the sums over the independent indices $g$ and $g^{\prime}$ in Eq. (3.3). We refer to the effect of $\mathcal{G}_{G \otimes G}$ as the local $G$-SSR.

The local $G$-SSR restricts the kinds of operations that the two parties can perform to local $G$-invariant operations $\mathcal{O}_{\mathrm{AB}}$ where

$$
\begin{aligned}
& \mathcal{O}_{\mathrm{AB}}\left\{\left[T_{\mathrm{A}}(g) \otimes T_{\mathrm{B}}\left(g^{\prime}\right)\right] \rho\left[T_{\mathrm{A}}^{\dagger}(g) \otimes T_{\mathrm{B}}^{\dagger}\left(g^{\prime}\right)\right]\right\} \\
& =T_{\mathrm{A}}(g) \otimes T_{\mathrm{B}}\left(g^{\prime}\right)\left\{\mathcal{O}_{\mathrm{AB}}[\rho]\right\} T_{\mathrm{A}}^{\dagger}(g) \otimes T_{\mathrm{B}}^{\dagger}\left(g^{\prime}\right) .
\end{aligned}
$$

for all $g, g^{\prime} \in G$. This class includes (but is not limited to) products of local operations $\mathcal{O}_{\mathrm{A}} \otimes \mathcal{O}_{\mathrm{B}}$, which could represent measurement outcomes. A wider class of allowed operations will be defined below. Moreover, any operation $\mathcal{O}_{\mathrm{AB}}$ which is local $G$-invariant is also global $G$-invariant, because Eq. (3.4) implies

$$
\mathcal{O}_{\mathrm{AB}}\left[T(g) \rho T^{\dagger}(g)\right]=T(g)\left\{\mathcal{O}_{\mathrm{AB}}[\rho]\right\} T^{\dagger}(g)
$$

for $T(g)=T_{\mathrm{A}}(g) \otimes T_{\mathrm{B}}(g)$.

\section{B. Globally-symmetric pure state $\rho^{\beta}$}

In this paper, we restrict our analysis to globally symmetric pure states:

$$
|\Psi\rangle\langle\Psi|=\mathcal{G}_{G}[|\Psi\rangle\langle\Psi|] .
$$

This requires $|\Psi\rangle$ to belong to a one-dimensional irrep of $G$. That is, using $\beta$ to label the irrep,

$$
T(g)|\Psi\rangle=\lambda^{\beta}(g)|\Psi\rangle \quad \forall g \in G
$$

where $T(g)$ is given by Eq. (3.1) and $\lambda^{\beta}(g)$ is the unitmodulus eigenvalue.

Let $G$ have $N_{G}$ distinct irreps $T^{\mu}(g)$ for $\mu=1,2, \cdots N_{G}$ and let Alice's operator $T_{\mathrm{A}}(g)$ in Eq. (3.1) decompose into $K_{\mathrm{A}}$ irreps as

$$
T_{\mathrm{A}}(g)=\bigoplus_{n=1}^{K_{\mathrm{A}}} T^{f_{\mathrm{A}}(n)}(g) \forall g \in G
$$

where

$$
f_{\mathrm{A}}(n) \in\left\{1,2, \cdots N_{G}\right\}
$$

labels an irrep for each $n$. The total number of irreps in $T_{\mathrm{A}}(g)$ can be written as $K_{\mathrm{A}}=\sum_{\mu=1}^{N_{G}} M_{\mathrm{A}}^{\mu}$, where $M_{\mathrm{A}}^{\mu}$ is the multiplicity (i.e. the number of copies) of irreps of type $T^{\mu}$. The irrep $T^{\mu}$ operates on the $D_{\mu}$-dimensional subspaces spanned by [3]

$$
\left\{\left|\mu, m_{\mu}, i\right\rangle: i=1,2, \cdots, D_{\mu}\right\} .
$$

The "charge" $\mu=1,2, \ldots N_{G}$ indexes the irreps $T^{\mu}$, the "flavor" $m_{\mu}=1,2, \ldots M_{\mathrm{A}}^{\mu}$ indexes the copy of the irrep $T^{\mu}$ in the above decomposition, the "color" $i=$ $1,2, \ldots D_{\mu}$ indexes an orthogonal basis set in which $T^{\mu}$ operates, and

$$
\left\langle\mu, m_{\mu}, i \mid \nu, m_{\nu}, j\right\rangle=\delta_{\mu, \nu} \delta_{m_{\mu}, m_{\nu}} \delta_{i, j}
$$

Let Bob's operator $T_{\mathrm{B}}(g)$ have a similar decomposition. To find the form of the global $G$-invariant states we need to consider pairs of conjugate irreps, that is pairs of irreps, say $T^{\mu}$ and $T^{\nu}$, whose tensor product $T^{\mu} \otimes T^{\nu}$, can be reduced to a direct sum involving a given 1dimensional irrep $T^{\beta}$ of $G$, i.e. $T^{\mu} \otimes T^{\nu} \cong T^{\beta} \oplus \ldots$ To do this we define $R^{\beta}$ to be the set of conjugate couples

$$
R^{\beta}=\left\{(\mu, \bar{\mu}): T^{\mu}(g)=C^{\mu} T^{\beta}(g)\left[T^{\bar{\mu}}(g)\right]^{*}\left(C^{\mu}\right)^{\dagger} \forall g \in G\right\}
$$

where $T^{\beta}$ is the given one-dimensional irrep and $C^{\mu}$ is a unitary operator. The entangled state

$$
\left|\psi_{m_{\mu}, m_{\bar{\mu}}}^{\mu, \beta}\right\rangle=\frac{1}{\sqrt{D_{\mu}}} \sum_{i, j} C_{i, j}^{\mu}\left|\mu, m_{\mu}, i\right\rangle \otimes\left|\bar{\mu}, m_{\bar{\mu}}, j\right\rangle
$$

for $(\mu, \bar{\mu}) \in R^{\beta}$, is an eigenstate of $T(g)$ with eigenvalue $\lambda^{\beta}(g)=T^{\beta}(g)$, and so it is globally symmetric. The proof of this result is given in Appendix 1.

The most general, pure, globally symmetric state for a given value of $\beta$ is given by

$$
\rho^{\beta}=\uplus\left|\Psi^{\beta}\right\rangle
$$

where

$$
\left|\Psi^{\beta}\right\rangle=\sum_{\mu} \sum_{m_{\mu}, m_{\bar{\mu}}} d_{m_{\mu}, m_{\bar{\mu}}}^{\mu}\left|\psi_{m_{\mu}, m_{\bar{\mu}}}^{\mu, \beta}\right\rangle
$$

for arbitrary coefficients $d_{m_{\mu}, m_{\bar{\mu}}}^{\mu}$ satisfying

$$
\sum_{m_{\mu}, m_{\bar{\mu}}}\left|d_{m_{\mu}, m_{\bar{\mu}}}^{\mu}\right|^{2}=1
$$

In the following we evaluate the effect of the $G$-SSR on the resources represented by this general state $\rho^{\beta}$. 


\section{Unconstrained entanglement of $\rho^{\beta}$}

We begin by evaluating the total entanglement in $\rho^{\beta}$, measured in terms of the entropy of entanglement, without the restriction of the $G$-SSR. It is convenient to factorize the representation into "flavor" (indexed by $m_{\mu}$ ) and "color" (indexed by $i$ ) subsystems as

$$
\left|\mu, m_{\mu}, i\right\rangle \equiv\left|\mu, m_{\mu}\right\rangle|\mu, i\rangle
$$

and rewrite the state in terms of states of the flavor and color subsystems as

$$
\left|\Psi^{\beta}\right\rangle=\sum_{\mu} \sqrt{P_{\mu}}\left|\varphi_{\mu}\right\rangle\left(\frac{1}{\sqrt{D_{\mu}}} \sum_{i, j} C_{i, j}^{\mu}|\mu, i\rangle \otimes|\bar{\mu}, j\rangle\right)
$$

where

$$
\begin{aligned}
\left|\varphi_{\mu}\right\rangle & =\sum_{m_{\mu}, m_{\mu}} \frac{d_{m_{\mu}, m_{\bar{\mu}}}^{\mu}}{\sqrt{P_{\mu}}}\left|\mu, m_{\mu}\right\rangle \otimes\left|\bar{\mu}, m_{\bar{\mu}}\right\rangle \\
P_{\mu} & =\sum_{m_{\mu}, m_{\bar{\mu}}}\left|d_{m_{\mu}, m_{\bar{\mu}}}^{\mu}\right|^{2} .
\end{aligned}
$$

Then taking the partial trace of $\rho^{\beta}$ over Bob's state space and making use of the unitarity of $C^{\mu}$ yields

$$
\operatorname{Tr}_{\mathrm{B}}\left(\rho^{\beta}\right)=\sum_{\mu} P_{\mu}\left(\biguplus_{k} \Lambda_{k}^{\mu}\left|\mathrm{A}_{k}^{\mu}\right\rangle\right) \otimes\left(\biguplus_{i} \frac{1}{\sqrt{D_{\mu}}}|\mu, i\rangle\right),
$$

where

$$
\biguplus_{i}\left|\psi_{i}\right\rangle \equiv \sum_{i} \uplus\left|\psi_{i}\right\rangle=\sum_{i}\left|\psi_{i}\right\rangle\left\langle\psi_{i}\right|,
$$

and we have used the Schmidt decomposition

$$
\left|\varphi_{\mu}\right\rangle=\sum_{k} \Lambda_{k}^{\mu}\left|\mathrm{A}_{k}^{\mu}\right\rangle \otimes\left|\mathrm{B}_{k}^{\mu}\right\rangle
$$

of the state of the bipartite flavor subsystem with

$$
\left\langle\mathrm{A}_{k}^{\mu} \mid \mathrm{A}_{l}^{\mu}\right\rangle=\left\langle\mathrm{B}_{k}^{\mu} \mid \mathrm{B}_{l}^{\mu}\right\rangle=\delta_{k, l} .
$$

Thus the entanglement is given by

$$
E\left(\rho^{\beta}\right)=-\sum_{\mu, k} P_{\mu}\left|\Lambda_{k}^{\mu}\right|^{2} \log \left(P_{\mu}\left|\Lambda_{k}^{\mu}\right|^{2}\right)+\sum_{\mu} P_{\mu} \log \left(D_{\mu}\right) .
$$

\section{Resources in $\rho^{\beta}$ under the local $G$-SSR}

\section{Entanglement accessible under local G-SSR}

The accessible entanglement in the state $\left|\Psi^{\beta}\right\rangle$ constrained by the local $G$-SSR is, according to [11], given by the total entanglement in the state [21].

$$
\mathcal{G}_{G \otimes G}\left[\rho^{\beta}\right]=\frac{1}{|G|^{2}} \biguplus_{g, g^{\prime} \in G}\left(\left[T_{\mathrm{A}}(g) \otimes T_{\mathrm{B}}\left(g^{\prime}\right)\right]\left|\Psi^{\beta}\right\rangle\right) .
$$

where $\mathcal{G}_{G \otimes G}$ is defined in Eq. (3.3). Using the unitarity of the matrices $C^{\mu}$ and the grand orthogonality theorem $[22]$

$$
\sum_{g \in G} T_{k, l}^{\mu}(g)\left[T_{n, m}^{\nu}(g)\right]^{*}=\frac{|G|}{D_{\mu}} \delta_{\mu, \nu} \delta_{k, n} \delta_{l, m}
$$

where $T_{i, j}^{\eta}=\left\langle\eta, m_{\eta}, i\left|T^{\eta}\right| \eta, m_{\eta}, j\right\rangle[22]$, yields

$$
\begin{aligned}
\mathcal{G}_{G \otimes G}\left[\rho^{\beta}\right]=\sum_{\mu} P_{\mu}\left(\uplus\left|\varphi_{\mu}\right\rangle\right) \\
\otimes \biguplus_{i, j}\left(\frac{1}{D_{\mu}}|\mu, i\rangle \otimes|\bar{\mu}, j\rangle\right)
\end{aligned}
$$

The entropy of this state is easily found using Eq. (3.26) and Eq. (3.27) to be

$$
S\left(\mathcal{G}_{G \otimes G}\left[\rho^{\beta}\right]\right)=H_{G \otimes G}^{(\mathrm{ch})}\left(\rho^{\beta}\right)+2 H_{G \otimes G}^{(\mathrm{co})}\left(\rho^{\beta}\right)
$$

where we have defined color and charge correlations

$$
\begin{aligned}
& H_{G \otimes G}^{(\mathrm{co})}\left(\rho^{\beta}\right) \equiv \sum_{\mu} P_{\mu} \log D_{\mu}, \\
& H_{G \otimes G}^{(\mathrm{ch})}\left(\rho^{\beta}\right) \equiv-\sum_{\mu} P_{\mu} \log P_{\mu} .
\end{aligned}
$$

We note that Alice (or, equivalently, Bob) can make a measurement of the charge without changing the amount of accessible entanglement, because the measurement commutes with all $G$-invariant operations $[9,11]$. The proof can be found in Appendix 2.

This local measurement of charge yields the value of $\mu$ with probability $P_{\mu}$, resulting in the pure entangled state $\left|\varphi_{\mu}\right\rangle$ of the flavor subsystem. The entanglement in the flavor subsystem is then the entropy $-\sum_{k}\left|\Lambda_{k}^{\mu}\right|^{2} \log \left(\left|\Lambda_{k}^{\mu}\right|^{2}\right)$ of Alice's reduced state, $\biguplus_{k}\left(\Lambda_{k}^{\mu}\left|\mathrm{A}_{k}^{\mu}\right\rangle\right)$. The corresponding state of the color subsystem in Eq. (3.28) is $\biguplus_{i, j}\left(|\mu, i\rangle \otimes|\bar{\mu}, j\rangle / D_{\mu}\right)$ which is clearly separable, and so the color subsystem makes no contribution to the entanglement. Averaging over all $\mu$ values gives the accessible entanglement $E_{G \otimes G}$ under local $G-S S R$ as

$$
E_{G \otimes G}\left(\rho^{\beta}\right)=E\left(\rho^{\beta}\right)-H_{G \otimes G}^{(\mathrm{co})}\left(\rho^{\beta}\right)-H_{G \otimes G}^{(\mathrm{ch})}\left(\rho^{\beta}\right) .
$$

The quantity $E_{G \otimes G}\left(\rho^{\beta}\right)$ in (3.32) represents the ability of the system under the local $G$-SSR to do "logical work" in the form of bipartite quantum information processing [15].

\section{Work extractable under local G-SSR and LOCC}

Just as in the unipartite case in the absence of the $G$ SSR, a bipartite state $\rho$ can be used to extract mechanical work locally at each site from local thermal reservoirs $[15,23]$. Only local operations and classical communication (LOCC) are allowed for the extraction process which 
results in a maximum amount of work $W_{\mathrm{L}}(\rho)$ being extracted in total. Oppenheim et al. [15] showed that the quantum correlations in the state $\rho$ reduce the amount of work that can be extracted in this way. Alternatively one could transmit the system at Alice's site to Bob's site through a dephasing channel and then extract the work locally at Bob's site. They showed that for pure states $\rho$ an equivalent amount of work $W_{\mathrm{L}}(\rho)$ is obtained if the dephasing channel produces a classically correlated state of minimum entropy. The allowed operations $\mathcal{Q}$ for this method are those that can be realized using local unitaries, local ancillas (whose extractable work must be subtracted off at the end) and transmission through the dephasing channel. That is [15]

$$
W_{\mathrm{L}}(\rho)=W(\widetilde{\mathcal{Q}}[\rho])
$$

where $\widetilde{\mathcal{Q}}$ is the optimum allowed operation that yields a classically-correlated state with minimal entropy $S(\widetilde{\mathcal{Q}}[\rho])$. For pure states there is a duality between abilities to do mechanical and "logical" work [15]:

$$
W(\rho)=W_{\mathrm{L}}(\rho)+E(\rho) .
$$

In particular, consider the locally extractable mechanical work from the pure state

$$
\sigma=\uplus\left(\sum_{n} \sqrt{p_{n}}\left|\phi_{n}\right\rangle_{\mathrm{AB}} \otimes\left|\chi_{n}\right\rangle_{\mathrm{AB}} \otimes\left|\psi_{n}\right\rangle_{\mathrm{AB}}\right)
$$

in the absence of the $G$-SSR. Here the $\left|\phi_{m}\right\rangle,\left|\chi_{m}\right\rangle$ and $\left|\psi_{m}\right\rangle$ represent states of three bipartite systems satisfying

$$
\left\langle\phi_{n} \mid \phi_{m}\right\rangle=\left\langle\chi_{n} \mid \chi_{m}\right\rangle=\left\langle\psi_{n} \mid \psi_{m}\right\rangle=\delta_{n, m} .
$$

From [15] the optimum operation $\widetilde{\mathcal{Q}}$ dephases $\sigma$ in its Schmidt basis; this can be carried out by first dephasing in the Schmidt basis of $\left\{\left|\chi_{n}\right\rangle \otimes\left|\psi_{n}\right\rangle\right\}$ followed by dephasing in the Schmidt basis of $\left\{\left|\phi_{n}\right\rangle\right\}$. Let the Schmidt bases be given by

$$
\left|x_{n}\right\rangle=\sum_{i} x_{n, i}\left|x_{n, i}\right\rangle
$$

where $x_{n, i}$ are the Schmidt coefficients and $\left|x_{n, i}\right\rangle$ are a set of orthonormal states, for $x$ being $\phi, \psi$ or $\chi$. The first step yields a state of the form

$$
\begin{aligned}
\sigma^{\prime}= & \sum_{n} p_{n}\left(\uplus\left|\phi_{n}\right\rangle_{\mathrm{AB}}\right) \\
& \otimes \biguplus_{i, j}\left(\chi_{n, i}\left|\chi_{n, i}\right\rangle_{\mathrm{AB}} \otimes \psi_{n, j}\left|\psi_{n, j}\right\rangle_{\mathrm{AB}}\right)
\end{aligned}
$$

and the second step yields a state of the form

$$
\begin{aligned}
\sigma^{\prime \prime}= & \sum_{n} p_{n} \biguplus_{k}\left(\phi_{n, k}\left|\phi_{n, k}\right\rangle_{\mathrm{AB}}\right) \\
& \otimes \biguplus_{i, j}\left(\chi_{n, i}\left|\chi_{n, i}\right\rangle_{\mathrm{AB}} \otimes \psi_{n, j}\left|\psi_{n, j}\right\rangle_{\mathrm{AB}}\right) .
\end{aligned}
$$

This can be reversibly transformed using the dephasing channel into

$$
\begin{aligned}
& \sum_{n} p_{n} \biguplus_{k}\left(\phi_{n, k}\left|\phi_{n, k}\right\rangle_{\mathrm{BB}}\right) \\
& \otimes \biguplus_{i, j}\left(\chi_{n, i}\left|\chi_{n, i}\right\rangle_{\mathrm{BB}} \otimes \psi_{n, j}\left|\psi_{n, j}\right\rangle_{\mathrm{BB}}\right),
\end{aligned}
$$

where the whole system is located at site B. The maximum amount of mechanical work that can be extracted from $\sigma$ locally at each site is equal to the maximum that can be extracted locally at site B from $\sigma^{\prime \prime}=\widetilde{\mathcal{Q}}[\sigma]$, as given in Eq. (3.33). We use this result below.

We now consider the local mechanical work $W_{G \otimes G-\mathrm{L}}(\rho)$ that is extractable from state $\rho$ under both the local $G$-SSR and the LOCC restrictions. This is given by

$W_{G \otimes G-\mathrm{L}}(\rho)=W_{\mathrm{L}}\left(\mathcal{G}_{G \otimes G}[\rho]\right)=W\left(\widetilde{\mathcal{Q}}_{G \otimes G}\left\{\mathcal{G}_{G \otimes G}[\rho]\right\}\right)$,

where $\widetilde{\mathcal{Q}}_{G \otimes G}$ is locally $G$-invariant.

We first evaluate $W\left(\widetilde{\mathcal{Q}}_{G \otimes G}\left\{\mathcal{G}_{G \otimes G}\left[\rho^{\beta}\right]\right\}\right)$. As $\mathcal{G}_{G \otimes G}\left[\rho^{\beta}\right]$ in Eq. (3.28) is equivalent in form to $\sigma^{\prime}$ in Eq. (3.38), the optimum operation $\widetilde{\mathcal{Q}}_{G \otimes G}$ is dephasing in the Schmidt basis of the flavor subsystem $\left\{\left|\varphi_{\mu}\right\rangle\right\}$ in Eq. (3.20). This can be done by making a local measurement in the Schmidt basis given in Eqs. (3.23) and (3.20). This operation can be shown to be local $G$-invariant as follows. For example, a local measurement by Alice that projects onto the Schmidt basis is described by the set of projection operators

$$
\widetilde{\Pi}_{k}=\left(\left|\mathrm{A}_{k}^{\mu}\right\rangle\left\langle\mathrm{A}_{k}^{\mu}\right| \otimes \mathbb{1}_{\mu}^{(\mathrm{co})}\right)_{\mathrm{A}} \otimes \mathbb{1}_{\mathrm{B}}
$$

for the same set of values of $k$ as in Eq. (3.23) and where $\mathbb{1}_{\mu}^{(\mathrm{co})}=\sum_{i=1}^{D_{\mu}}|\mu, i\rangle\langle\mu, i|$ projects onto the color subsystem. Recalling the decomposition Eq. (3.8) and noting that the irrep $T^{\mu}(g)$ acts on the corresponding color subsystem only, we find

$$
\begin{aligned}
& {\left[T_{\mathrm{A}}(g) \otimes T_{\mathrm{B}}\left(g^{\prime}\right)\right] \widetilde{\Pi}_{k}} \\
& \quad=T_{\mathrm{A}}(g) \otimes T_{\mathrm{B}}\left(g^{\prime}\right)\left[\left(\left|\mathrm{A}_{k}^{\mu}\right\rangle\left\langle\mathrm{A}_{k}^{\mu}\right| \otimes \mathbb{1}_{\mu}^{(\mathrm{co})}\right)_{\mathrm{A}} \otimes \mathbb{1}_{\mathrm{B}}\right] \\
& \quad=\left[\left|\mathrm{A}_{k}^{\mu}\right\rangle\left\langle\mathrm{A}_{k}^{\mu}\right| \otimes T^{\mu}(g)\right]_{\mathrm{A}} \otimes T_{\mathrm{B}}\left(g^{\prime}\right) \\
& \quad=\left[\left(\left|\mathrm{A}_{k}^{\mu}\right\rangle\left\langle\mathrm{A}_{k}^{\mu}\right| \otimes \mathbb{1}_{\mu}^{(\mathrm{co})}\right)_{\mathrm{A}} \otimes \mathbb{1}_{\mathrm{B}}\right]\left[T_{\mathrm{A}}(g) \otimes T_{\mathrm{B}}\left(g^{\prime}\right)\right] \\
& \quad=\widetilde{\Pi}_{k}\left[T_{\mathrm{A}}(g) \otimes T_{\mathrm{B}}\left(g^{\prime}\right)\right] .
\end{aligned}
$$

That is, projection by $\widetilde{\Pi}_{k}$ is a locally $G$-invariant operation according to Eq. (3.4). The average result of Alice's 
measurement gives the desired optimal dephasing, i.e.

$$
\begin{aligned}
& \widetilde{\mathcal{Q}}_{G \otimes G}\left\{\mathcal{G}_{G \otimes G}\left[\rho^{\beta}\right]\right\} \\
& =\sum_{k} p_{k} \widetilde{\Pi}_{k} \rho^{\beta} \widetilde{\Pi}_{k} \\
& =\biguplus_{\mu, k, i, j}\left(\frac{1}{D_{\mu}} \sqrt{P_{\mu}} \Lambda_{k}^{\mu}\left|\mathrm{A}_{k}^{\mu}\right\rangle \otimes\left|\mathrm{B}_{k}^{\mu}\right\rangle \otimes|\mu, i\rangle \otimes|\bar{\mu}, j\rangle\right),
\end{aligned}
$$

where $p_{k}=\operatorname{Tr}\left(\widetilde{\Pi}_{k} \rho^{\beta}\right)$.

Now using Eq. (3.33) the locally extractable work is found to be

$$
\begin{aligned}
W_{G \otimes G-\mathrm{L}}\left(\rho^{\beta}\right) & =W\left(\widetilde{\mathcal{Q}}_{G \otimes G}\left\{\mathcal{G}_{G \otimes G}\left[\rho^{\beta}\right]\right\}\right) \\
& =\ln (D)-S\left(\widetilde{\mathcal{Q}}_{G \otimes G}\left\{\mathcal{G}_{G \otimes G}\left[\rho^{\beta}\right]\right\}\right) \\
& =\log D-\left[E\left(\rho^{\beta}\right)+H_{G \otimes G}^{(\operatorname{co})}\left(\rho^{\beta}\right)\right]
\end{aligned}
$$

The global symmetry of the pure state $\rho^{\beta}$ ensures that

$$
W_{G}\left(\rho^{\beta}\right)=W\left(\mathcal{G}_{G}\left[\rho^{\beta}\right]\right)=W\left(\rho^{\beta}\right)=\log D
$$

and so the locally extractable work can be written as

$$
W_{G \otimes G-\mathrm{L}}\left(\rho^{\beta}\right)=W\left(\rho^{\beta}\right)-\left[E\left(\rho^{\beta}\right)+H_{G \otimes G}^{(\mathrm{co})}\left(\rho^{\beta}\right)\right] .
$$

Finally, using Eq. (3.34) we can rearrange Eq. (3.47) as

$$
W_{G \otimes G-\mathrm{L}}\left(\rho^{\beta}\right)=W_{\mathrm{L}}\left(\rho^{\beta}\right)-H_{G \otimes G}^{(\mathrm{co})}\left(\rho^{\beta}\right)
$$

which shows that the reduction in $W_{\mathrm{L}}$ due to the local $G$-SSR is manifest in the mixing in the color subsystems.

\section{Shared asymmetry with respect to local G-SSR}

Eqs. (3.32) and (3.47) show that under the local $G$-SSR the duality between logical and local mechanical work in Eq. (3.34) is broken, i.e. $W \neq W_{G \otimes G-\mathrm{L}}+E_{G \otimes G}$. Just as in the unipartite case, the lack of a reference system results in the loss of the ability to do work. In this globallysymmetric bipartite case what is lacking is a shared reference system. For a globally symmetric system to act as a shared reference there must be correlations (quantum or classical) between the asymmetries for each party. These correlations are unaffected by a global transformation $\mathcal{G}$, but are destroyed by local mixing $\mathcal{G}_{G \otimes G}$. This suggests the natural entropic measure for the ability of such a system to act as a shared reference is

$$
A_{G \otimes G}^{(\mathrm{sh})}(\rho) \equiv S\left(\mathcal{G}_{G \otimes G}\left\{\mathcal{G}_{G}[\rho]\right\}\right)-S\left(\mathcal{G}_{G}[\rho]\right)
$$

which we call the shared asymmetry. Notice that here $\rho$ is an arbitrary state which is not necessarily pure nor globally symmetric. By shared we mean that both Alice and Bob have access to this type of asymmetry for unlocking the resources represented by $\rho$ at their sites. The global asymmetry $A_{G}[\rho]$ of the state is not, in itself, useful for this purpose. To eliminate the effects of global asymmetry we have defined $A_{G \otimes G}^{(\mathrm{sh})}(\rho)$ in Eq. (3.49) in terms of the globally-symmetric state $\mathcal{G}_{G}[\rho]$. The result is that $A_{G \otimes G}^{(\mathrm{sh})}(\rho)$ is equal to the increase in entropy due to the local $G$-SSR only. For the U(1) case the refbit [14] has $A_{G \otimes G}^{(\mathrm{sh})}=1$, as one would like.

In analogy with $A_{G}$ for the unipartite case, we now show that $A_{G \otimes G}^{(\mathrm{sh})}$ similarly quantifies the resource of acting as a shared reference system for arbitrary states $\rho$. First we note that

$$
\begin{aligned}
\mathcal{G}_{G \otimes G}\left\{\mathcal{G}_{G}[\rho]\right\} & =\frac{1}{|G|^{2}} \sum_{g, g^{\prime} \in G} \frac{1}{|G|} \sum_{g^{\prime \prime} \in G}\left[T\left(g^{\prime}\right) \otimes T(g)\right]\left[T\left(g^{\prime \prime}\right) \otimes T\left(g^{\prime \prime}\right)\right] \rho\left[T^{\dagger}\left(g^{\prime \prime}\right) \otimes T^{\dagger}\left(g^{\prime \prime}\right)\right]\left[T^{\dagger}\left(g^{\prime}\right) \otimes T^{\dagger}(g)\right] \\
& =\frac{1}{|G|^{2}} \sum_{g, g^{\prime} \in G} \frac{1}{|G|} \sum_{g^{\prime \prime} \in G}\left[T\left(g^{\prime} \circ g^{\prime \prime}\right) \otimes T\left(g \circ g^{\prime \prime}\right)\right] \rho\left[T^{\dagger}\left(g^{\prime} \circ g^{\prime \prime}\right) \otimes T^{\dagger}\left(g \circ g^{\prime \prime}\right)\right] \\
& =\frac{1}{|G|^{2}} \sum_{g, g^{\prime} \in G} \frac{1}{|G|} \sum_{h \in G}\left[T\left(g^{\prime} \circ g^{-1} \circ h\right) \otimes T(h)\right] \rho\left[T^{\dagger}\left(g^{\prime} \circ g^{-1} \circ h\right) \otimes T^{\dagger}(h)\right] \\
& =\frac{1}{|G|} \sum_{h^{\prime} \in G} \frac{1}{|G|} \sum_{h \in G}\left[T\left(h^{\prime}\right) T(h) \otimes T(h)\right] \rho\left[T^{\dagger}(h) T^{\dagger}\left(h^{\prime}\right) \otimes T^{\dagger}(h)\right] \\
& =\frac{1}{|G|} \sum_{h^{\prime} \in G} \frac{1}{|G|} \sum_{h \in G}\left[T\left(h^{\prime}\right) \otimes \mathbb{1}\right][T(h) \otimes T(h)] \rho\left[T^{\dagger}(h) \otimes T^{\dagger}(h)\right]\left[T^{\dagger}\left(h^{\prime}\right) \otimes \mathbb{1}\right] \\
& =\mathcal{G}_{G \otimes\{e\}}\left\{\mathcal{G}_{G}[\rho]\right\}=\mathcal{G}_{\{e\} \otimes G}\left\{\mathcal{G}_{G}[\rho]\right\}
\end{aligned}
$$

where $h=g \circ g^{\prime \prime}, h^{\prime}=g^{\prime} \circ g^{-1}$ and $\{e\}$ is the group

containing only the identity element so that, for example,

$$
\mathcal{G}_{G \otimes\{e\}}[\rho]=\frac{1}{|G|} \sum_{g \in G}\left[T_{\mathrm{A}}(g) \otimes \mathbb{1}_{\mathrm{B}}\right] \rho\left[T_{\mathrm{A}}^{\dagger}(g) \otimes \mathbb{1}_{\mathrm{B}}\right] .
$$


Similarly we can show

$$
\mathcal{G}_{G \otimes G}\left\{\mathcal{G}_{G}[\rho]\right\}=\mathcal{G}_{\{e\} \otimes G}\left\{\mathcal{G}_{G}[\rho]\right\}
$$

and this means that the shared asymmetry may be written equivalently as

$$
A_{G \otimes G}^{(\mathrm{sh})}(\rho)=S\left(\mathcal{G}_{G \otimes G}[\rho]\right)-S\left(\mathcal{G}_{G}[\rho]\right) .
$$

The properties of the entropy function show $A_{G \otimes G}^{(\mathrm{sh})}$ has the following two properties:

(i) $A_{G \otimes G}^{(\mathrm{sh})}(\rho) \geq 0$;

(ii) $A_{G \otimes G}^{(\mathrm{sh})}(\rho)=0$ iff $\mathcal{G}_{G \otimes G}[\rho]=\mathcal{G}_{G}[\rho]$.

For global $G$-invariant states $\rho^{\beta}$ we have $\mathcal{G}_{G}\left[\rho^{\beta}\right]=\rho^{\beta}$ and so the second property becomes

$(\text { ii })^{\prime} A_{G \otimes G}^{(\mathrm{sh})}\left(\rho^{\beta}\right)=0$ iff $\mathcal{G}_{G \otimes G}\left[\rho^{\beta}\right]=\rho^{\beta}$, or, equivalently, iff $\mathcal{G}_{G \otimes\{e\}}\left[\rho^{\beta}\right]=\mathcal{G}_{\{e\} \otimes G}\left[\rho^{\beta}\right]=\rho^{\beta}$.

A third property is

(iii) $A_{G \otimes G}^{(\mathrm{sh})}(\rho)$ is non-increasing on average under local $G$-invariant local operations and classical communication (LOCC),

which is analogous to the third property of $A_{G}$. We define local $G$-invariant LOCC as those LOCC that are allowed by the local $G$-SSR or, equivalently, those that satisfy Eq. (3.4). These include products of local operations of the form $\mathcal{O}_{\mathrm{A}} \otimes \mathcal{O}_{\mathrm{B}}$. For classical communication to be permitted under the $G$-SSR, the information must be carried by physical processes that are permitted by the $G$-SSR. This can be done, for example, by using a $G$-SSR-free system as the carrier, which we assume is the case. The class of LOCC is a subset of the class of separable operations [24]. It is straight-forward to show, using the same reasoning as in Ref. [24], that every local $G$-invariant LOCC operation is a local $G$-invariant separable operation. So to prove the third property it is sufficient to show that $A_{G \otimes G}^{(\mathrm{sh})}(\rho)$ is non-increasing under local $G$-invariant separable operations of the type

$$
\left\{\mathcal{O}_{\mathrm{A}, i} \otimes \mathcal{O}_{\mathrm{B}, j}: i=1,2, \ldots, j=1,2, \ldots,\right\}
$$

where $\mathcal{O}_{\mathrm{A}, i} \otimes \mathcal{O}_{\mathrm{B}, j}$ satisfies Eq. (3.4). We therefore wish to show that

$$
A_{G \otimes G}^{(\mathrm{sh})}(\rho) \geq \sum_{i, j} P_{i, j} A_{G \otimes G}^{(\mathrm{sh})}\left(\rho_{i, j}\right)
$$

where

$$
\begin{aligned}
\rho_{i, j} & =\frac{1}{P_{i, j}}\left(\mathcal{O}_{\mathrm{A}, i} \otimes \mathcal{O}_{\mathrm{B}, j}\right)[\rho] \\
P_{i, j} & =\operatorname{Tr}\left[\left(\mathcal{O}_{\mathrm{A}, i} \otimes \mathcal{O}_{\mathrm{B}, j}\right) \rho\right],
\end{aligned}
$$

or equivalently, from Eq. (3.49),

$$
\begin{aligned}
& S\left(\mathcal{G}_{G \otimes G}\left\{\mathcal{G}_{G}[\rho]\right\}\right)-S\left(\mathcal{G}_{G}[\rho]\right) \\
& \geq \sum_{i, j} P_{i, j}\left[S\left(\mathcal{G}_{G \otimes G}\left\{\mathcal{G}_{G}\left[\rho_{i, j}\right]\right\}\right)-S\left(\mathcal{G}_{G}\left[\rho_{i, j}\right]\right)\right] .
\end{aligned}
$$

We note that according to Eqs. (3.4) and (3.5) each element in the set in Eq. (3.54) is also global $G$-invariant, and so

$$
\mathcal{G}_{G}\left\{\left(\mathcal{O}_{\mathrm{A}, i} \otimes \mathcal{O}_{\mathrm{B}, j}\right)[\rho]\right\}=\left(\mathcal{O}_{\mathrm{A}, i} \otimes \mathcal{O}_{\mathrm{B}, j}\right)\left\{\mathcal{G}_{G}[\rho]\right\}
$$

This means we can interchange the twirl and measurement operations in Eq. (3.56). Let $\varrho \equiv \mathcal{G}_{G}[\rho]$ and

$$
\varrho_{i, j} \equiv \frac{1}{P_{i, j}}\left(\mathcal{O}_{\mathrm{A}, i} \otimes \mathcal{O}_{\mathrm{B}, j}\right)\left\{\mathcal{G}_{G}[\rho]\right\}=\mathcal{G}_{G}\left[\rho_{i, j}\right]
$$

We can now rewrite Eq. (3.56) as

$$
S\left(\mathcal{G}_{G \otimes G}[\varrho]\right)-S(\varrho) \geq \sum_{i, j} P_{i, j}\left[S\left(\mathcal{G}_{G \otimes G}\left[\varrho_{i, j}\right]\right)-S\left(\varrho_{i, j}\right)\right]
$$

which is in the same form as Eq. (2.16). The same arguments which follow Eq. (2.16) can be used to show that the right hand side of Eq. (3.59), and thus $A_{G \otimes G}^{(\mathrm{sh})}(\rho)$, is non-increasing under local $G$-invariant separable operations, and by implication, that the third property is therefore valid.

The total amount of extractable work under the local $G$-SSR is the sum of the logical work and the locally extracted mechanical work, i.e. $\left(W_{G \otimes G-\mathrm{L}}+E_{G \otimes G}\right)$. Using Eqs. (3.29), (3.32) and (3.45) we find

$$
\begin{aligned}
& W_{G \otimes G-\mathrm{L}}\left(\rho^{\beta}\right)+E_{G \otimes G}\left(\rho^{\beta}\right) \\
& \quad=\log D-H_{G \otimes G}^{(\mathrm{ch})}\left(\rho^{\beta}\right)-2 H_{G \otimes G}^{(\mathrm{co})}\left(\rho^{\beta}\right) \\
& \quad=\log D-S\left(\mathcal{G}_{G \otimes G}\left[\rho^{\beta}\right]\right) .
\end{aligned}
$$

This total is equivalent to just the mechanical work $W_{G \otimes G}\left(\rho^{\beta}\right)$, where we define

$$
W_{G \otimes G}(\rho) \equiv \log D-S\left(\mathcal{G}_{G \otimes G}[\rho]\right)
$$

for arbitrary states $\rho$ (which are not necessarily globally symmetric). The physical interpretation of $W_{G \otimes G}(\rho)$ is that under the local $G$-SSR, $\mathcal{G}_{G \otimes G}(\rho)$ is the effective state of the system which can be transferred locally to SSRfree ancillas at each site. Once the transfer is done the amount of work that can be extracted globally from a thermal reservoir (i.e. without the LOCC restriction) using the ancillas is $W_{G \otimes G}(\rho)$. Eqs. (3.60) and (3.61) show that an equivalent physical interpretation of $W_{G \otimes G}(\rho)$ is that it is the total extractable work, both logical and mechanical, that can be extracted under the local $G$-SSR and LOCC. This result leads to the fourth and final property that

(iv) the shared asymmetry is an achievable upper bound on the synergy of the total extractable work $W_{G \otimes G}$.

This follows from the following theorem. 
Theorem 3. The synergy of the total work $W_{G \otimes G}$ under the local G-SSR is bounded by the shared asymmetry, i.e.

$$
\Upsilon\left(W_{G \otimes G}^{(\mathrm{tot})} ; \rho_{1}, \rho_{2}\right) \leq \min \left\{A_{G \otimes G}^{(\mathrm{sh})}\left(\rho_{1}\right), A_{G \otimes G}^{(\mathrm{sh})}\left(\rho_{2}\right)\right\} .
$$

The upper bound is achievable in the sense of theorem 2.

We omit the proof, which has the same form as that of Theorem 2. The achievability follows from the existence of bipartite globally symmetric states $|\Psi\rangle$ such that

$$
\left\langle\Psi\left|T_{\mathrm{A}}(g)^{\dagger} T_{\mathrm{A}}\left(g^{\prime}\right)\right| \Psi\right\rangle=\delta_{g, g^{\prime}} .
$$

Thus we have identified three resources that emerge in a bipartite setting under a $G$-SSR: the locally extractable mechanical work $W_{G \otimes G-\mathrm{L}}$, the accessible entanglement or logical work $E_{G \otimes G}$, and the shared asymmetry $A_{G \otimes G}^{(\mathrm{sh})}$. Finally, we show that, for globally $G$-invariant states, there is a triality relation between them, generalizing the duality (2.37) from the unipartite setting. A straightforward calculation gives

$$
A_{G \otimes G}^{(\mathrm{sh})}\left(\rho^{\beta}\right)=2 H_{G \otimes G}^{(\mathrm{co})}\left(\rho^{\beta}\right)+H_{G \otimes G}^{(\mathrm{ch})}\left(\rho^{\beta}\right),
$$

which, together with Eq. (3.32) and Eq. (3.47), then gives the main result of this paper

$$
W_{G}\left(\rho^{\beta}\right)=W_{G \otimes G-\mathrm{L}}\left(\rho^{\beta}\right)+E_{G \otimes G}\left(\rho^{\beta}\right)+A_{G \otimes G}^{(\mathrm{sh})}\left(\rho^{\beta}\right) .
$$

\section{Local asymmetry with respect to local G-SSR}

We defined the shared asymmetry of state $\rho$ in Eq. (3.49) as the extra entropy generated by the local $G$-SSR acting on the state $\mathcal{G}_{G}[\rho]$, i.e. $A_{G \otimes G}^{(\mathrm{sh})}(\rho)=$ $S\left(\mathcal{G}_{G \otimes G}\left\{\mathcal{G}_{G}[\rho]\right\}\right)-S\left(\mathcal{G}_{G}[\rho]\right)$. It is interesting to consider the entropy generated by the local $G$-SSR acting on the state $\rho$ itself. For this purpose we define

$$
A_{G \otimes G}^{(\mathrm{lo})}(\rho) \equiv S\left(\mathcal{G}_{G \otimes G}[\rho]\right)-S(\rho),
$$

which we call the local asymmetry of $\rho . A_{G \otimes G}^{(\mathrm{lo})}$ is related to the shared $A_{G \otimes G}^{(\mathrm{sh})}$ and global $A_{G}(\rho)$ asymmetries by

$$
\begin{aligned}
& A_{G \otimes G}^{(\mathrm{sh})}(\rho)=A_{G \otimes G}^{(\mathrm{lo})}\left(\mathcal{G}_{G}[\rho]\right) \\
& A_{G \otimes G}^{(\mathrm{lo})}(\rho)=A_{G \otimes G}^{(\mathrm{sh})}(\rho)+A_{G}(\rho) .
\end{aligned}
$$

As $A_{G \otimes G}^{(\mathrm{sh})}(\rho) \geq 0$ and $A_{G}(\rho) \geq 0$ then clearly

$$
0 \leq A_{G \otimes G}^{(\mathrm{sh})}(\rho) \leq A_{G \otimes G}^{(\mathrm{lo})}(\rho) .
$$

The local asymmetry $A_{G \otimes G}^{(\mathrm{lo})}(\rho)$ is the asymmetry of $\rho$ with respect to the local $G$-SSR which restricts our knowledge of the state to $\mathcal{G}_{G \otimes G}[\rho]$. It is clearly related to the total extractable work $W_{G \otimes G}(\rho)$ that is represented by the state $\mathcal{G}_{G \otimes G}[\rho]$. Indeed from Eqs. (2.5), (3.66) and (3.61) we find

$$
W(\rho)=W_{G \otimes G}(\rho)+A_{G \otimes G}^{(\mathrm{lo})}(\rho) .
$$

We can now list the properties of the local asymmetry as

(i) $A_{G \otimes G}^{(\mathrm{lo})}(\rho) \geq 0$;

(ii) $A_{G \otimes G}^{(\mathrm{lo})}(\rho)=0$ iff

$$
\mathcal{G}_{G \otimes\{e\}}[\rho]=\rho
$$

and

$$
\mathcal{G}_{\{e\} \otimes G}[\rho]=\rho
$$

(iii) $A_{G \otimes G}^{(\mathrm{lo})}(\rho)$ is non-increasing on average under local $G$-invariant LOCC; and

(iv) $A_{G \otimes G}^{(\mathrm{lo})}(\rho)$ is an achievable upper bound on the synergy of the extractable work $W_{G \otimes G}$ under the local $G$-SSR restriction.

Once again the first property follows from the properties of the entropy function. The proof of the third property is a minor modification of the proof of the third property of the shared asymmetry $A_{G \otimes G}^{(\mathrm{sh})}$. Similarly, the proof of the fourth property is of the same form as that of the fourth property of the asymmetry $A_{G}$. We leave these proofs for the interested reader.

The second property can be proved as follows. We note that the conditions Eqs. (3.71) and (3.72) taken together imply $\mathcal{G}_{G \otimes G}[\rho]=\rho$ for which $A_{G \otimes G}^{(\text {lo })}(\rho)=0$ according to Eq. (3.66) and so the conditions are sufficient. Also the concavity of the entropy function yields $S\left(\mathcal{G}_{G \otimes G}[\rho]\right) \geq S\left(\mathcal{G}_{G \otimes\{e\}}[\rho]\right) \geq S(\rho)$. Thus $A_{G \otimes G}^{(\mathrm{lo})}(\rho)=0$ implies $S\left(\mathcal{G}_{G \otimes\{e\}}[\rho]\right)=S(\rho)$ and hence $\mathcal{G}_{G \otimes\{e\}}[\rho]=\rho$. By a similar argument, $A_{G \otimes G}^{(\mathrm{lo})}(\rho)=0$ implies that $\mathcal{G}_{\{e\} \otimes G}[\rho]=\rho$. The conditions are therefore necessary as well.

Either condition Eq. (3.71) or Eq. (3.72) is sufficient for $\mathcal{G}_{G \otimes G}[\rho]=\mathcal{G}_{G}[\rho]$ and thus sufficient for $A_{G \otimes G}^{(\mathrm{sh})}[\rho]=$ 0. But both these conditions are not necessary for $A_{G \otimes G}^{(\mathrm{sh})}[\rho]=0$. This means there is a wider class of states for which $A_{G \otimes G}^{(\mathrm{lo})}[\rho] \neq 0$ than for $A_{G \otimes G}^{(\mathrm{sh})}[\rho] \neq 0$.

Finally, Eqs. (3.61) and (3.70) together yield

$$
W\left(\rho^{\beta}\right)=W_{G \otimes G-\mathrm{L}}\left(\rho^{\beta}\right)+E_{G \otimes G}\left(\rho^{\beta}\right)+A_{G \otimes G}^{(\mathrm{lo})}\left(\rho^{\beta}\right)
$$

which is consistent with Eq. (3.65) on recalling Eq. (3.68) and Eq. (2.37) and the fact that $A_{G}\left(\rho^{\beta}\right)=0$ for the globally symmetric state $\rho^{\beta}$. 


\section{DISCUSSION}

In this paper we have quantified the ability of a system to act as a reference system and ameliorate the effect of the superselection rule $G$-SSR induced by $G$. Our approach is to express the reference-frame ability of a system in terms of a physical quantity, namely, in terms of how the system can increase the amount of work that can extracted from a thermal reservoir. To do this we introduced the quantity $\Upsilon$ in Eq. (2.22), which we call the synergy of two systems. The work synergy is the extra amount of work that is extractable using the two systems collectively compared to the total amount of work extractable using the systems separately. Theorem 2 shows that this quantity is bounded above by the asymmetry $A_{G}$ with respect to symmetry group $G$ of each system, a result which elevates the asymmetry of a system to a resource for overcoming the restrictions of the $G$-SSR. We used the same approach for bipartite systems where we found (Theorem 3) that the synergy bounds the shared asymmetry $A_{G \otimes G}^{(\mathrm{sh})}$.

Our results can be arranged in terms of a hierarchy of increasing restrictions, from global $G$-SSR, global and local $G$-SSR and finally global and local $G$-SSR and LOCC. At each level of restriction we find that the resources reappear in new forms. For example, under the global $G$ SSR Eq. (2.37) shows that the unconstrained extractable work $W$ splits into two new resources of extractable work $W_{G}$ and asymmetry $A_{G}$, i.e.

$$
W(\rho)=W_{G}(\rho)+A_{G}(\rho)
$$

for arbitrary states $\rho$. Next under global and local $G$-SSR we find from Eqs. (4.1), (3.70) and (3.68) that the extractable work $W_{G}$ further splits into a more constrained extractable work $W_{G \otimes G}$ and a new asymmetry $A_{G \otimes G}^{(\mathrm{sh})}$, i.e.

$$
W_{G}(\rho)=W_{G \otimes G}(\rho)+A_{G \otimes G}^{(\mathrm{sh})}(\rho)
$$

also for arbitrary states $\rho$. Finally under global and local $G$-SSR and LOCC we found

$$
W_{G \otimes G}\left(\rho^{\beta}\right)=W_{G \otimes G-\mathrm{L}}\left(\rho^{\beta}\right)+E_{G \otimes G}\left(\rho^{\beta}\right)
$$

for globally symmetric states $\rho^{\beta}$. These results are summarized in Table I. A different ordering of the constraints, where LOCC is applied first followed by the global $G$-SSR and then the local $G$-SSR leads to the results in Table II.

Our relations Eqs. (2.37) and (3.65) show the mutually competing nature of the mechanical, logical and asymmetry resources represented by a state. They are analogous to the particle-wave duality in the following sense. Asymmetry with respect to the group $G=\{g\}$ can be thought of as a generalized measure of localization in that the most asymmetric pure state is transformed into an orthogonal state by the group elements $g$ which is analogous to moving a particle from one distinct path to

\begin{tabular}{|c|c|c|}
\hline Constraints & Resources & State \\
\hline- & $W$ & $\rho$ \\
$G$ & $W=W_{G}+A_{G}$ & $\rho$ \\
$\left.\begin{array}{c}G \& G \otimes G \\
G, G \otimes G \\
\& \mathrm{~L}\end{array}\right\}$ & $W_{G}=W_{G \otimes G}+A_{G \otimes G}^{(\mathrm{sh})}$ & $\rho$ \\
& & \\
\hline
\end{tabular}

TABLE I: Hierarchy of constraints and resources for classes of states where $\rho$ represents an arbitrary state and $\rho^{\beta}$ represents a pure $G$-invariant state.

\begin{tabular}{|c|c|c|}
\hline Constraints & Resources & State \\
\hline- & $W$ & $\rho$ \\
$\mathrm{L}$ & $W=W_{\mathrm{L}}+E$ & $\rho$ \\
$\mathrm{L} \& G$ & $W_{G}=W_{G-\mathrm{L}}+E_{G}$ & $\rho^{\beta}$ \\
$\mathrm{L}, G \&$ \\
$\left.\begin{array}{c}G \otimes G\end{array}\right\}$ & $W_{G-\mathrm{L}}+E_{G}$ & $\rho^{\beta}$ \\
\hline
\end{tabular}

TABLE II: Hierarchy for a different ordering of the constraints. The equation in the third row is obtained directly from the second row using the fact that $W_{G}\left(\rho^{\beta}\right)=W\left(\rho^{\beta}\right)$, $W_{G-\mathrm{L}}\left(\rho^{\beta}\right)=W_{\mathrm{L}}\left(\rho^{\beta}\right)$ and $E_{G}\left(\rho^{\beta}\right)=E\left(\rho^{\beta}\right)$ for $G$-invariant states $\rho^{\beta}$.

another in a which-way experiment. On the other hand, extractable work under the $G$-SSR measures the invariance of a state to the group action and can be thought of as a measure of the system's ability to display interference. Our relation Eq. (2.37) between asymmetry and extractable work can then be seen to express a tradeoff between generalized measures of localization and interference. This connection has been explored elsewhere [25].

SSRs are ubiquitous in quantum physics where, for example, spatial orientation is limited by a $\mathrm{SU}(n)$-SSR and optical phase is limited by a U(1)-SSR. In the presence of SSRs, quantum states require sufficient asymmetry in their attendant reference systems in order to be useful. Moreover, a comparison of the relative efficiencies of classical and quantum algorithms needs to account for the total amount of resources needed in each case. Quantum reference systems are clearly a resource that needs to be tallied and so, in this sense, our results pave the way for evaluating the full cost of resources needed for quantum information processing. They also open up a new direction of research in the study of SSRs and reference systems.

We thank M. Plenio and S.D. Bartlett for discussions. This work was supported by the Australian Research Council, the Queensland State Government and the Leverhulme Trust of the UK. 


\section{APPENDIX}

\section{Proof of Eq. (3.13)}

We show that $\left|\psi_{m_{\mu}, m_{\bar{\mu}}}^{\mu, \beta}\right\rangle$ in Eq. (3.13) is globally symmetric. From Eqs. (3.8), (3.12) and (3.13), the scalar nature of $T^{\beta}$ and the unitarity of the operators $C^{\mu}$ and $T^{\mu}(g)$ we find that

$$
\begin{aligned}
T(g)\left|\psi_{m_{\mu}, m_{\bar{\mu}}}^{\mu, \beta}\right\rangle & =\bigoplus_{i=1}^{K_{\mathrm{A}}} \bigoplus_{j=1}^{K_{\mathrm{B}}} T^{f_{\mathrm{A}}(i)}(g) \otimes T^{f_{\mathrm{B}}(j)}(g)\left|\psi_{m_{\mu}, m_{\bar{\mu}}}^{\mu, \beta}\right\rangle \\
& =T^{\mu}(g) \otimes T^{\bar{\mu}}(g)\left|\psi_{m_{\mu}, m_{\bar{\mu}}}^{\mu, \beta}\right\rangle \\
& =\left[C^{\mu} T^{\beta}(g)\left[T^{\bar{\mu}}(g)\right]^{*}\left(C^{\mu}\right)^{\dagger}\right] \otimes T^{\bar{\mu}}(g)\left|\psi_{m_{\mu}, m_{\bar{\mu}}}^{\mu, \beta}\right\rangle \\
& =\frac{1}{\sqrt{D_{\mu}}} \sum_{i, j} C_{i, j}^{\mu} C^{\mu} T^{\beta}(g)\left[T^{\bar{\mu}}(g)\right]^{*}\left(C^{\mu}\right)^{\dagger}\left|\mu, m_{\mu}, i\right\rangle \otimes T^{\bar{\mu}}(g)\left|\bar{\mu}, m_{\bar{\mu}}, j\right\rangle \\
& =\frac{1}{\sqrt{D_{\mu}}} \sum_{i, j, k, l, n, p} C_{i, j}^{\mu} C_{n, l}^{\mu} T^{\beta}(g)\left[T_{l, k}^{\bar{\mu}}(g)\right]^{*}\left(C_{i, k}^{\mu}\right)^{*} T_{p, j}^{\bar{\mu}}(g)\left|\mu, m_{\mu}, n\right\rangle \otimes\left|\bar{\mu}, m_{\bar{\mu}}, p\right\rangle \\
& =\frac{1}{\sqrt{D_{\mu}}} \sum_{n, p} C_{n, l}^{\mu} T^{\beta}(g)\left|\mu, m_{\mu}, n\right\rangle \otimes\left|\bar{\mu}, m_{\bar{\mu}}, p\right\rangle \\
& =T^{\beta}(g)\left|\psi_{m_{\mu}, m_{\bar{\mu}}}^{\mu, \beta}\right\rangle \\
& =\lambda^{\beta}(g)\left|\psi_{m_{\mu}, m_{\bar{\mu}}}^{\mu, \beta}\right\rangle
\end{aligned}
$$

where $\lambda^{\beta}(g)$ is an eigenvalue of unit modulus. Thus

$$
T(g)\left(\uplus\left|\psi_{m_{\mu}, m_{\bar{\mu}}}^{\mu, \beta}\right\rangle\right) T^{\dagger}(g)=\uplus\left|\psi_{m_{\mu}, m_{\bar{\mu}}}^{\mu, \beta}\right\rangle
$$

which completes the proof that $\left|\psi_{m_{\mu}, m_{\bar{\mu}}}^{\mu, \beta}\right\rangle$ is globally symmetric.

\section{Measurement of charge under the local $G$-SSR}

We show here that a local measurement of charge is locally $G$-invariant and thus it is an allowed operation under the local $G$-SSR. Let the projection operators onto the flavor and color subsystems be

$$
\begin{aligned}
\mathbb{1}_{\mu}^{(\mathrm{f})} & =\sum_{m_{\mu}=1}^{M^{\mu}}\left|\mu, m_{\mu}\right\rangle\left\langle\mu, m_{\mu}\right| \\
\mathbb{1}_{\mu}^{(\mathrm{co})} & =\sum_{i=1}^{D_{\mu}}|\mu, i\rangle\langle\mu, i|,
\end{aligned}
$$

respectively, where the states $|\mu, i\rangle$ and $\left|\mu, m_{\mu}\right\rangle$ are defined by Eq. (3.17) and $D_{\mu}$ is the dimension and $M^{\mu}$ is the multiplicity of the irrep labeled by $\mu$. We note from Eq. (3.8) that the operator $\left(\mathbb{1}_{\mu}^{(\mathrm{fl})} \otimes \mathbb{1}_{\mu}^{(\mathrm{co})}\right)$ "picks out" the irrep $T^{\mu}$ in the following sense

$$
\begin{aligned}
T(g)\left(\mathbb{1}_{\mu}^{(\mathrm{fl})} \otimes \mathbb{1}_{\mu}^{(\mathrm{co})}\right) & =T^{\mu}(g)\left(\mathbb{1}_{\mu}^{(\mathrm{fl})} \otimes \mathbb{1}_{\mu}^{(\mathrm{co})}\right) \\
& =\left(\mathbb{1}_{\mu}^{(\mathrm{fl})} \otimes \mathbb{1}_{\mu}^{(\mathrm{co})}\right) T^{\mu}(g) \\
& =\left(\mathbb{1}_{\mu}^{(\mathrm{fl})} \otimes \mathbb{1}_{\mu}^{(\mathrm{co})}\right) T(g)
\end{aligned}
$$

and so $\left[T(g),\left(\mathbb{1}_{\mu}^{(\mathrm{fl})} \otimes \mathbb{1}_{\mu}^{(\mathrm{co})}\right)\right]=0$. A local measurement by Alice that projects onto the charge $\mu$ is described by the set of projection operators

$$
\Pi_{\mu}=\left(\mathbb{1}_{\mu}^{(\mathrm{fl})} \otimes \mathbb{1}_{\mu}^{(\mathrm{co})}\right)_{\mathrm{A}} \otimes \mathbb{1}_{\mathrm{B}}
$$

for $\mu=1,2, \ldots, N_{G}$ where $N_{G}$ is the number of irreps of $G$ and subscripts A and B refer to operators acting on Alice's and Bob's subsystems, respectively. We find that

$$
\begin{aligned}
& T_{\mathrm{A}}(g) \otimes T_{\mathrm{B}}\left(g^{\prime}\right)\left(\Pi_{\mu} \rho \Pi_{\mu}\right) T_{\mathrm{A}}^{\dagger}(g) \otimes T_{\mathrm{B}}^{\dagger}\left(g^{\prime}\right) \\
& =\Pi_{\mu}\left[T_{\mathrm{A}}(g) \otimes T_{\mathrm{B}}\left(g^{\prime}\right)\right] \rho\left[T_{\mathrm{A}}^{\dagger}(g) \otimes T_{\mathrm{B}}^{\dagger}\left(g^{\prime}\right)\right] \Pi_{\mu}
\end{aligned}
$$

and so the local projection measurement of charge given by $\left\{\Pi_{\mu}: \mu=1,2, \ldots, N_{G}\right\}$ is locally $G$-invariant according to Eq. (3.4). 
[1] G.C. Wick, A.S. Wightman and E.P. Wigner, Phys. Rev. 80, 101 (1952).

[2] Y.Aharonov and L.Susskind, Phys. Rev. 155, 1428 (1967).

[3] A. Kitaev, D. Mayers, J. Preskill, Phys. Rev. A 69, 052326 (2004); arXiv:quant-ph/0310088.

[4] S.D. Bartlett, T. Rudolph, R.W. Spekkens, Rev. Mod. Phys. 79, 555 (2007); arXiv:quant-ph/0610030.

[5] J. Eisert, T. Felbinger, P. Papadopoulos, M. B. Plenio, and M. Wilkens, Phys. Rev. Lett. 84, 1611 (2000).

[6] S. J. Jones, H. M. Wiseman, S. D. Bartlett, J. A. Vaccaro, D. T. Pope, Phys. Rev. A 74, 062313 (2006); arXiv:quant-ph/0608056v2.

[7] See, e.g., E. Bagan, M. Baig, and R. Munoz-Tapia, Phys. Rev. Lett. 87, 257903 (2001); T. Rudolph and L. Grover, Phys. Rev. Lett. 91, 217905 (2003); and references therein.

[8] S.D. Bartlett, T. Rudolph, and R.W. Spekkens, Phys. Rev. Lett. 91, 027901 (2003); ibid., Phys. Rev. A 70, 032307 (2004).

[9] H.M. Wiseman and J.A. Vaccaro, Phys. Rev. Lett. 91, 097902 (2003).

[10] M.R. Dowling, A.C. Doherty, and H.M. Wiseman, Phys. Rev. A 73, 052323 (2006); arXiv:quant-ph/0601079v2.

[11] S.D. Bartlett and H.M. Wiseman, Phys. Rev. Lett. 91, 097903 (2003)

[12] J.A. Vaccaro, F. Anselmi and H.M. Wiseman, Int. J. Quant. Inf. 1, 427 (2003); arXiv:quant-ph/0311028v2.

[13] N. Schuch, F. Verstraete, and J.I. Cirac, Phys. Rev. Lett. 92, 087904 (2004).

[14] S.J. van Enk, Phys. Rev. A 71, 032339 (2005); arXiv:quant-ph/0410083v3.
[15] J. Oppenheim, M. Horodecki, P. Horodecki and R. Horodecki, Phys. Rev. Lett. 89, 180402 (2002).

[16] Where we give expressions for discrete groups, the corresponding analysis for compact Lie groups is obtained by replacing summations with appropriate integrals etc.

[17] It is interesting to note that Aberg [26] has recently introduced a measure of the degree of superposition which has the same form as our asymmetry measure $A_{G}(\rho)$.

[18] J. von Neumann, Translated by R.T. Beyer Mathematical Foundations of Quantum Mechanics (Princeton University Press, Princeton, 1955) Ch 5, Section 2.

[19] B. Schumacher, M. Westmoreland and W.K. Wootters, Phys. Rev. Lett. 76, 3452 (1996).

[20] K. Jacobs, J. Math. Phys. 47, 012102 (2006); arXiv:quant-ph/0412006v2

[21] Note also that $\left[\mathcal{G}_{\mathrm{A}} \otimes \mathcal{G}_{\mathrm{B}}\right](\mathcal{G} \rho)=\left[\mathcal{G}_{\mathrm{A}} \otimes \mathcal{G}_{\mathrm{B}}\right] \rho$.

[22] See, e.g., H.F. Jones, Groups, representations and physics, (Adam Hilger, Bristol, 1990).

[23] M. Horodecki, K. Horodecki, P. Horodecki, R. Horodecki, J. Oppenheim, A. Sen(De), and U. Sen, Phys. Rev. Lett. 90, $100402(2003)$

[24] C.H. Bennett, D.P. DiVincenzo, C.A. Fuchs, T. Mor, E. Rains, P.W. Shor, J.A. Smolin and W.K. Wootters, Phys. Rev. A 59, 1070 (1999).

[25] J.A. Vaccaro, in Proceedings of the 8th International Conference on Quantum Communication, Measurement and Computing, edited by O.Hirota, J.H. Shiparo and M.Sasaki (NICT Press, Tokyo, 2007), p. 421.

[26] J. Aberg, "Quantifying Superposition", arXiv:quant$\mathrm{ph} / 0612146 \mathrm{v} 1$ 